\title{
BIOLOGIA REPRODUTIVA DE OLIGOSARCUS JENYNSII (GÜNTHER) (CHARACIFORMES, CHARACIDAE) DA LAGOA DAS CUSTÓDIAS, TRAMANDAÍ, RIO GRANDE DO SUL, BRASIL
}

\author{
Clarice B. Fialho ${ }^{1}$ \\ Lucia C. Schifino ${ }^{2}$ \\ José Roberto Verani ${ }^{3}$
}

\begin{abstract}
REPRODUCTIVE BIOLOGY OF OLIGOSARCUS JENYNSII (GÜNTHER) (CHARACiformes, Characidae) From Custódias Lagoon, Tramandaí, Rio Grande do Sul, BrazIL. The Custódias lagoon has a great importance in the Tramandai system, because it works as reproduction area, feeding and nursery grounds to several fish species. The aim of this work is the study of aspects related to the reproduction of Oligosarcus jenynsii (Günther, 1864) as such as reproductive period, sexual ratio, fecundity and size of the first gonadal maturation. The specimens were sampled monthly from October/1994 to October/1995. For each sex, maturation curves were established, based on the monthly variation of the gonadossomatic index mean values, as well as, the relative frequencies of the sexual maturity stages. Breeding seasons took place during the winter and spring. The sex ratio was significanthly different from 1:1, with more females than male in almost all month. The species had a average fecundity of 9.694 ovocytes. The estimated size of first sexual maturation was $114 \mathrm{~mm}$ for male and $131 \mathrm{~mm}$ for females.

KEY WORDS. Characiformes, Oligosarcus jenynsii, fecundity, reproduction
\end{abstract}

A lagoa das Custódias, que faz parte do Sistema Lagunar de Tramandaí, Rio Grande do Sul, caracteriza-se por apresentar uma ictiofauna constituída por representantes tanto de água doce, como marinha. Sendo assim, é de grande importância para os peixes costeiros, pois serve como área de reprodução, criadouro natural e alimentação, constituindo-se em um local para manutenção e proteção temporal do ciclo de muitas espécies.

Oligosarcus jenynsii (Günther, 1864), popularmente conhecida como tambicu, branca ou peixe-cachorro, é uma das espécies mais constantes e abundantes nas lagoas costeiras do litoral norte do Rio Grande do Sul. A espécie tem como distribuição geográfica os arroios, rios e lagoas da região costeira e da planície interior do Rio Grande do Sul estendendo-se, também, pelos territórios do Uruguai e Argentina (MENEZES 1987).

1) Departamento de Zoologia, Laboratório de Ictiologia, Universidade Federal do Rio Grande do Sul. Avenida Paulo Gama, 90046-900 Porto Alegre, Rio Grande do Sul, Brazil.

2) Curso de Pós-graduação em Ecologia, Universidade Federal do Rio Grande do Sul. Avenida Bento Gonçalves 9500, 91501-970 Porto Alegre, Rio Grande do Sul, Brazil.

3) Departamento de Hidrobiologia, Universidade Federal de São Carlos. Caixa Postal 676, 13560-000 São Carlos, São Paulo, Brazil.

Revta bras. Zool. 15 (3): 775 - 782, 1998 
Estudos referentes à biologia e ecologia deste grupo são escassos, podendo ser citados os trabalhos de MENEZES (1969); FREYRE et al. (1984); HARO \& GUTIERREZ (1985); AQUINO (1991) HARTZ \& BARBIERI (1995) e HARTZ et al. (1997). Segundo MENEZES (1969) esta espécie possui um hábito carnívoro, alimentando-se, principalmente, de outros peixes e insetos e que habita, preferencialmente, os ambientes lênticos. Apresenta um dimorfismo sexual quanto ao crescimento, sendo que as fêmeas alcançam tamanhos maiores do que os machos (HARTZ \& BARBIERI 1995). Quanto aos aspectos reprodutivos os únicos trabalhos conhecidos são os de IWASZKIW et al. (1983) e HARTZ et al. (1997).

O presente trabalho tem como objetivos estudar os aspectos relacionados à reprodução de $O$. jenynsii na lagoa das Custódias, tais como: época de reprodução, proporção sexual, tipo de desova, fecundidade absoluta e relativa e tamanho de primeira maturação gonadal.

\section{MATERIAL E MÉTODOS}

A lagoa das Custódias, situada no litoral norte do Estado do Rio Grande do Sul, faz parte da Bacia Hidrográfica do Rio Tramandaí ( $\left.30^{\circ} 01^{\prime} \mathrm{S}, 50^{\circ} 10^{\prime} \mathrm{W}\right)$ sendo a primeira do conjunto de lagoas interligadas que se dispõem paralelamente ao mar e que recebem influência salina, via laguna do Armazém. É uma lagoa rasa $(1,10 \mathrm{~m}$ de profundidade média), alongada no sentido noroeste-sudeste, ocupando uma superfície de $9,58 \mathrm{Km}^{2}$.

Os exemplares foram coletados, mensalmente, de outubro de 1994 a outubro de 1995. Para tal, foram utilizadas quatro baterias de redes de espera de diversas malhas ( $1,0 \mathrm{a} 4,0 \mathrm{~cm}$ entrenós adjacentes) dispostas perpendicular à margem ficando as mesmas expostas por um período de 24 horas, com revisões a cada 6 horas. De cada indivíduo foram tomadas as medidas de comprimento total (Lt), em mm, peso total (Wt), em g, peso das gônadas (wg), em g, e, identificados o sexo e os estádios de maturação gonadal. Estes foram caracterizados levando-se em consideração os aspectos macroscópicos tais como, cor, vascularização, flacidez, tamanho em relação à cavidade abdominal e a visualização dos ovócitos. Desta forma, foram definidos cinco estádios para machos e fêmeas: imaturo (imat), em maturação (emat), maduro (mad), semi-esgotado (sesg) e esgotado (esg).

O período reprodutivo, para machos e fêmeas, foi estabelecido através das distribuições mensais das freqüências relativas de cada estádio de maturação gonadal, bem como da variação mensal dos valores médios do índice gonadossomático (IGS) segundo SANTOS (1978). Este foi calculado como o percentual que o peso das gônadas representou no peso total.

A proporção sexual foi determinada através das distribuições mensais das freqüências relativas de machos e fêmeas ao longo do período de estudo. Utilizou-se o teste do "qui-quadrado" para a verificação de eventuais diferenças significativas nestas proporções estimadas em relação à esperada de 1:1.

Para a estimativa da fecundidade foram selecionadas as gônadas que encontravam-se no estádio maduro as quais foram colocadas em solução de Gilson modificada (VAZZOLER 1981) até o total desprendimento dos ovócitos da parede 
ovariana. Após, foram lavadas e conservadas em álcool 70\%. Para a contagem dos ovócitos, foi utilizada uma pipeta do tipo Stempell de $2,5 \mathrm{ml}$ retirando-se três subamostragens de um volume total de $250 \mathrm{ml}$. A fecundidade absoluta (F) foi estimada através do modelo: $\mathrm{N}=\mathrm{nV} / \mathrm{v}$, onde $(\mathrm{N})$ número total de ovócitos; $(\mathrm{n})$ número médio de ovócitos vitelinados obtido nas subamostragens; (V) volume total da solução; (v) volume da subamostra. Para a obtenção da fecundidade relativa os dados de fecundidade absoluta foram relacionados com o comprimento total (Lt), peso total (Wt) e peso das gônadas (wg), através do método dos mínimos quadrados.

O tamanho de primeira maturação gonadal (Lpm) foi estimado segundo SANTOS (1978). Este tamanho foi considerado como aquele em que $50 \%$ dos indivíduos da população estão aptos à reprodução.

\section{RESULTADOS}

Foram amostrados 379 indivíduos (240 fêmeas e 139 machos), com variação de comprimento total de 92 a $228 \mathrm{~mm}$ para os machos e de 97 a $243 \mathrm{~mm}$, para as fêmeas.

Os resultados das relações lineares entre os pesos das gônadas (wg) e peso total (Wt), para cada estádio de maturação gonadal, são encontrados na tabela I, confirmando, assim, o uso do índice gonadossomático. Os valores médios mensais do índice gonadossomático foram lançados em gráfico mostrando sua variação durante o período de estudo (Fig. 1). Observa-se que $O$. jenynsii, na lagoa das Custódias, reproduz-se entre os meses de junho a outubro, correspondendo ao período de inverno e primavera. Confirmou-se através do teste $X^{2}(\alpha=0,05)$ que a reprodução da espécie é periódica anual, revelando haver uma relação significativa entre a época do ano e as freqüências observadas dos estádios de maturação gonadal. A variação mensal das frequêencias relativas dos estádios de maturação gonadal revelou que a maior incidência de indivíduos maduros ocorreu nos meses de junho, julho e agosto, evidenciando, desta forma, o período reprodutivo (Tab. II). Observa-se, ainda, que os indivíduos imaturos foram amostrados durante os meses de novembro, dezembro, janeiro e março, meses estes subseqüentes à época reprodutiva.

Tabela I. Dados referentes às regressōes lineares entre o peso das gônadas (wg) e o peso total (Wt) e valores médios do indice gonadossomático (IGS), por estádio de maturação gonadal, para machos e fêmeas de Oligosarcus jenynsii durante o período de outubro de 1994 a outubro de 1995.

\begin{tabular}{|c|c|c|c|c|c|c|}
\hline \multirow{2}{*}{ Estádio } & \multicolumn{2}{|c|}{$w g=b . w t$} & \multicolumn{2}{|c|}{$r$} & \multicolumn{2}{|c|}{$\overline{I G S}$} \\
\hline & Macho & Fèmea & Macho & Fêmea & Macho & Fêmea \\
\hline Imaturo & $w g=0,0040 . w t$ & $w g=0,0034 . w t$ & 0,79 & 0,80 & 0,23 & 0,32 \\
\hline Em maturaçăo & $w g=0,0102 . w t$ & $w g=0,0311 . w t$ & 0,83 & 0,80 & 0,92 & 2,79 \\
\hline Maduro & $w g=0,0174 . W t$ & $w g=0,0724 . w t$ & 0,79 & 0,71 & 1,79 & 7,02 \\
\hline Semi-esgotado & $w g=0,0073 . w t$ & $w g=0,0207 . w t$ & 0,87 & 0,69 & 0,69 & 2,01 \\
\hline Esgotado & $w g=0,0023 . w t$ & $w g=0,0067 . w t$ & 0,89 & 0,81 & 0,15 & 0,64 \\
\hline
\end{tabular}

(b) Valor do coeficiente de regressão, (r) coeficiente de correlação linear de Pearson. 

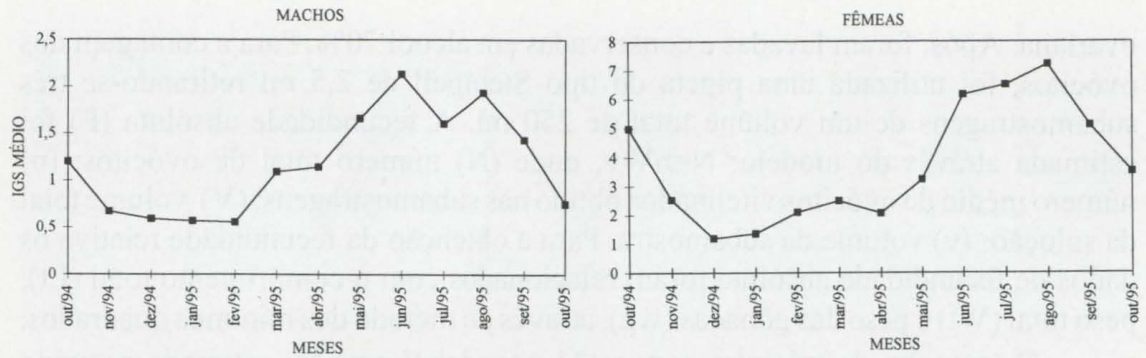

Fig. 1. Variação mensal dos valores médios do indice gonadossomático (IGS) para machos e fêmeas de Oligosarcus jenynsii durante o período de outubro de 1994 a outubro de 1995.

Tabela II. Distribuição mensal das freqüências relativas dos estádios de maturação gonadal para machos e fêmeas de Oligosarcus jenynsii durante o período de outubro de 1994 a outubro de 1995.

\begin{tabular}{|c|c|c|c|c|c|c|c|c|c|c|}
\hline \multirow{2}{*}{ Meses } & \multicolumn{2}{|c|}{ Imaturo } & \multicolumn{2}{|c|}{ Em maturação } & \multicolumn{2}{|c|}{ Maduro } & \multicolumn{2}{|c|}{ Semi-esgotado } & \multicolumn{2}{|c|}{ Esgotado } \\
\hline & Macho & Fèmea & Macho & Fêmea & Macho & Fêmea & Macho & Fêmea & Macho & Fêmea \\
\hline Outubro/94 & 11,1 & 13,0 & 16,7 & 0,0 & 66,7 & 52,0 & 5,5 & 32,0 & 0,0 & 3,0 \\
\hline Novembro/95 & 36,4 & 33,4 & 0,0 & 0,0 & 0,0 & 11,1 & 63,6 & 44,4 & 0,0 & 11,1 \\
\hline Dezembro/95 & 0,0 & 17,6 & 0,0 & 5,9 & 5,5 & 5,9 & 66,7 & 35,3 & 27,8 & 35,3 \\
\hline Janeiro/95 & 0,0 & 16,6 & 0,0 & 0,0 & 0,0 & 0,0 & 50,0 & 41,7 & 16,7 & 41,7 \\
\hline Fevereiro/95 & 0,0 & 0,0 & 66,7 & 38,4 & 33,3 & 15,4 & 0,0 & 23,1 & 0,0 & 23,1 \\
\hline Março/95 & 0,0 & 4,8 & 22,2 & 47,6 & 44,5 & 23,8 & 33,3 & 19,0 & 0,0 & 4,8 \\
\hline Abril/95 & 0,0 & 0,0 & 73,3 & 47,1 & 20,0 & 23,5 & 0,0 & 17,6 & 6,7 & 11,8 \\
\hline Maio/95 & 0,0 & 0,0 & 0,0 & 50,0 & 90,9 & 45,8 & 9,1 & 4,2 & 0,0 & 0,0 \\
\hline Junho/95 & 0,0 & 0,0 & 14,3 & 25,0 & 85,7 & 75,0 & 0,0 & 0,0 & 0,0 & 0,0 \\
\hline Julho/95 & 0,0 & 0,0 & 55,5 & 38,9 & 44,5 & 61,1 & 0,0 & 0,0 & 0,0 & 0,0 \\
\hline Agosto/95 & 0,0 & 0,0 & 20,0 & 28,6 & 80,0 & 64,3 & 0,0 & 7,1 & 0,0 & 0,0 \\
\hline Setembro/95 & 0,0 & 6,7 & 15,4 & 0,0 & 38,5 & 0,0 & 46,1 & 53,3 & 0,0 & 40,0 \\
\hline Outubro/95 & 25,0 & 0,0 & 0,0 & 0,0 & 0,0 & 30,8 & 75,0 & 61,5 & 0,0 & 7,7 \\
\hline
\end{tabular}

A fecundidade absoluta apresentou uma valor médio de 9694 ovócitos, com valores mínimos e máximos de 2625 à 13720 , respectivamente. Os resultados da fecundidade relativa são apresentados na figura 2. Observou-se que o número de ovócitos aumentou em função das variáveis envolvidas, sendo do tipo potencial para comprimento total (Fig. 2a) e linear para peso total e peso das gônadas (Figs 2b,c, respectivamente).

Para $O$. jenynsii da lagoa das Custódias, observou-se que a proporção sexual foi significativamente diferente de 1:1, com predomínio de fêmeas em quase todos os meses, com exceção de novembro, dezembro, abril, julho e setembro onde houve proporcionalidade entre machos e fêmeas (Fig. 3).

O tamanho de primeira maturação gonadal foi estimado em $114 \mathrm{~mm}$ para machos e $131 \mathrm{~mm}$ para as fêmeas. A partir de, aproximadamente, $145 \mathrm{~mm}$ e 210 $\mathrm{mm}$ todos os machos e fêmeas, respectivamente, estão aptos à reprodução (Fig. 4).

\section{DISCUSSÃO}

A desova de $O$. jenynsii, da lagoa das Custódias, tendo início nos meses de inverno onde a temperatura da água é a mais baixa, também foi observada por HARTZ et al. (1997) na lagoa Caconde, para a mesma espécie, e BAzzoli \& 
(a)

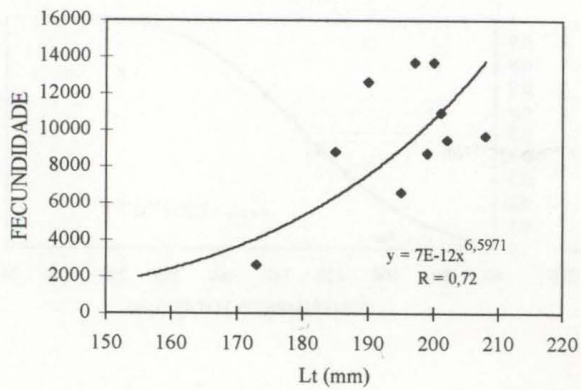

(b)

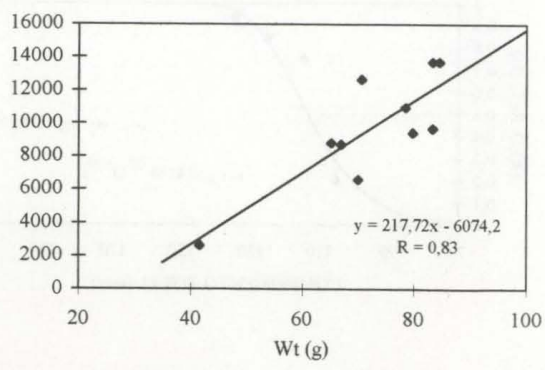

(c)

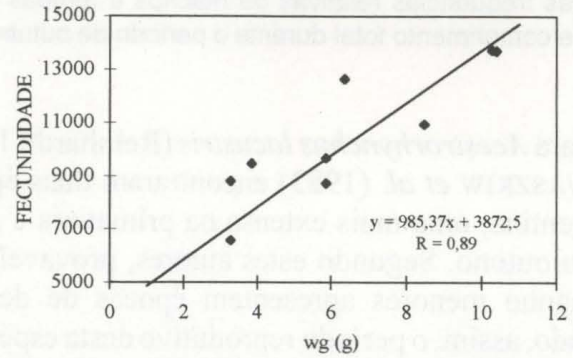

Fig. 2. Relação entre a fecundidade absoluta e o comprimento total (Lt) (a), peso total (Wt) (b) e peso das gônadas (wg) (c) para fêmeas de Oligosarcus jenynsii durante o período de outubro de 1994 a outubro de 1995.

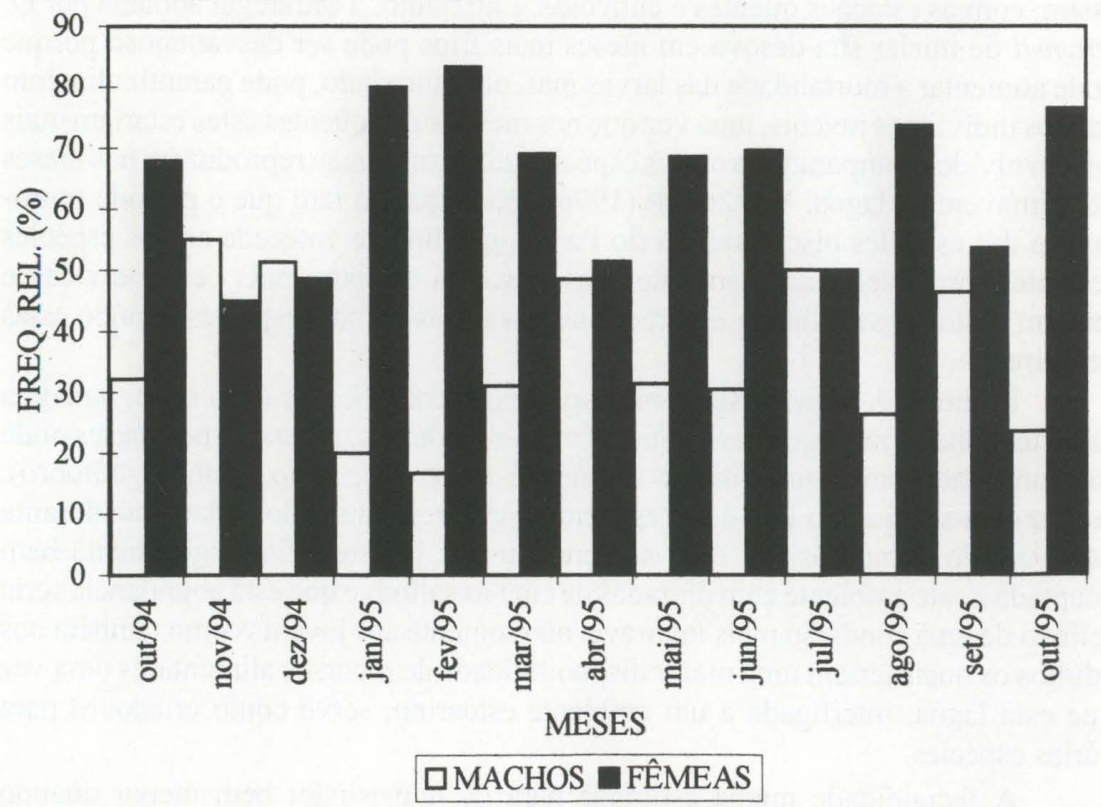

Fig. 3. Distribuição mensal das freqüências relativas de machos e fêmeas de Oligosarcus jenynsii durante o período de outubro de 1994 a outubro de 1995. 
MACHOS

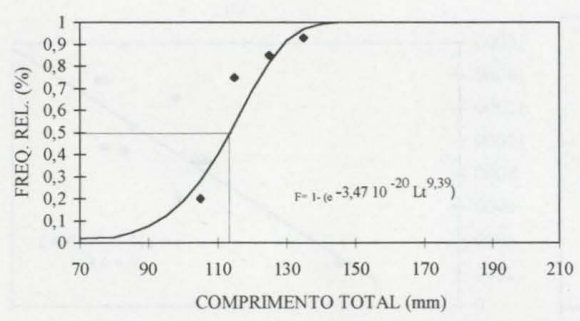

FÊMEAS

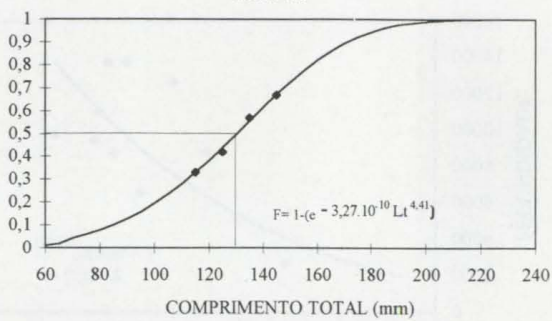

Fig. 4. Distribuição das freqüências relativas de machos e fêmeas adultos de Oligosarcus jenynsii por classes de comprimento total durante o período de outubro de 1994 a outubro de 1995.

GODINHO (1991) para Acestrorhynchus lacustris (Reinhardt, 1874) no Reservatório de Três Marias. IWASZKIW et al. (1983) encontraram duas épocas de desova para $O$. jenynsii na Argentina, uma mais extensa na primavera e princípios de verão e outra mais curta no outono. Segundo estes autores, provavelmente, os indivíduos de classes de tamanho menores apresentem épocas de desovas diferentes das maiores, prolongando, assim, o período reprodutivo desta espécie que possui desova única. VAZZOLER \& MENEZES (1992), em sua síntese sobre o comportamento reprodutivo de Characiformes, relatam que a maioria das espécies pertencentes a esta ordem, no sul do Brasil, reproduzem-se na primavera e verão coincidindo, assim, com as estações quentes e chuvosas. Entretanto, a estratégia adotada por $O$. jenynsii de iniciar sua desova em meses mais frios pode ser desvantajoso porque pode aumentar a mortalidade das larvas mas, por outro lado, pode garantir alimento para os indivíduos juvenis, uma vez que nos meses subseqüentes estes estariam mais desenvolvidos comparados a outras espécies de peixes que se reproduzem nos meses de primavera na lagoa. VAZZOLER (1996) aponta para o fato que o período reprodutivo das espécies piscívoras do rio Paraná geralmente antecede ao das espécies restantes, provavelmente para que suas larvas já estejam mais desenvolvidas e tenham maior possibilidade de predar as larvas das demais espécies quando estas eclodirem.

Embora $O$. jenynsii seja uma espécie característica de água doce, na lagoa das Custódias, a mesma esteve entre as mais abundantes, inclusive nos meses onde foi constatado haver uma maior salinidade (fevereiro, maio, junho e outubro). Acrescenta-se a isso, o fato desta espécie estar se reproduzindo justamente durante este período. Sendo assim, é de se acreditar que já esteja fisiologicamente bem adaptada a este ambiente com entradas de cunhas salinas e que esta abundância seria reflexo de uma condição mais favorável não somente aos juvenis como também aos adultos os quais teriam uma maior disponibilidade de recursos alimentares uma vez que esta lagoa, interligada a um ambiente estuarino, serve como criadouro para várias espécies.

A fecundidade média estimada para $O$. jenynsii foi bem menor quando comparada com as obtidas por IWASZKIW et al. (1983) e HARTZ et al. (1997) para a mesma espécie só que em ambientes de água doce. Ao compararmos $O$. jenynsii 
com outros Characiformes (VAZZOLER \& MENEZES 1992), esta pode ser categorizada como uma espécie que apresenta uma fecundidade intermediária entre os representantes do grupo, isto é, maior que as espécies que apresentam cuidados parentais como Hoplias malabaricus (Bloch, 1794) e menor que as espécies que apresentam migrações reprodutivas como Salminus maxillosus (Valenciennes, 1840) e Prochilodus scrofa (Steindachner, 1881). Segundo WootTon (1991), a relação entre a fecundidade relativa e as variáveis peso e comprimento é do tipo potencial. Isto foi constatado para $O$. jenynsii quanto ao comprimento total. Porém, as funções que melhor se ajustaram às relações entre fecundidade e peso total e peso das gônadas foram lineares, fato este também comprovado por HARTZ et al. (1997). IWASZKIW et al. (1983), por outro lado, encontraram relações potenciais para comprimento e peso totais e linear somente para o peso das gônadas.

Em caracídeos, geralmente o que se evidencia é o predomínio de fêmeas (SVERLiJ \& Ros 1986; MORAES et al. 1988; SANTOS et al. 1991; FONTOURA et al. 1993, entre outros). Neste trabalho, também se verificou haver uma maior proporção de fêmeas, principalmente no período que antecede à reprodução. HARTZ et. al. (1997) para $O$. jenynsii da lagoa Caconde observaram que a proporção sexual de 1:1 foi mantida quando considerado o total de exemplares coletados, porém quando analisadas as proporções mensais, verificaram haver um maior predomínio de fêmeas. As variações encontradas na proporção sexual de $O$. jenynsii na lagoa das Custódias podem estar relacionadas ao grau de exigência diferenciada que cada sexo apresenta por determinados recursos ambientais encontrados neste local.

AGRADECIMENTOS. À Sandra Maria Hartz pelas críticas e sugestões e Cristiano Machado Silveira pelo auxilio em campo. Ao Centro de Ecologia/UFRGS pelo apoio às saídas de campo e uso de suas dependências. A CAPES pela concessão das bolsas de doutorado e mestrado.

\section{REFERÊNCIAS BIBLIOGRÁFICAS}

AQuINO, A.E. 1991. Alimentación de Oligosarcus jenynsii (Günther, 1864) (Osteichthyes, Characidae) en el embalse el Cadillal (Tucuman, Argentina). Biologia Acuática, La Plata, 15 (2): 178-179.

BAzzoli, N. \& H.P. GodinHo. 1991. Reproductive biology of Acestrorhynchus lacustris (Reinhardt, 1874) (Pisces, Characidae) from Três Marias reservoir, Brazil. Zool. Anz., Leipzig, 226 (5/6): 285-297.

FonTOURA, N.F.; C.R. WECK; M.X. DE T. LUTZ \& W.R. KocH. 1993. Dinâmica populacional da ictiofauna da lagoa Fortaleza, Cidreira, Rio Grande do Sul. I. Astyanax alburnus (Hensel, 1870) (Teleostei, Charciformes, Characidae). Biociências, Porto Alegre, 1 (1): 77-94.

FreYre, L.R.; O.H. PADIN \& L.C. Protogino. 1984. Metabolismo energetico de peces dulceacuícolas. IV. Oligosarcus jenynsii (Pisces, Characidae). Limnobios La Plata, 2 (8): 629-634.

HARO, J.G. \& M. GUTIÉRREZ. 1985. Alimentación de Oligosarcus jenynsii (Günther) (Pisces, Characidae) en el Lago San Roque (Cordoba, Argentina). Rev. Assoc. Cienc. Nat. del Litoral, Buenos Aires, 16 (2): 227-235. 
HARTZ, S.M. \& G. BARBIERI. 1995. Crescimento do peixe-cachorro, Oligosarcus jenynsii (Günther, 1864), na lagoa Caconde, Rio Grande do Sul, Brasil (Teleostei, Characidae). Bol. Inst. Pesca, São Paulo, 22 (2): 33-40.

HARTZ, S.M.; F. Vilella \& G. BARBIERI. 1997. Reproduction dynamics of Oligosarcus jenynsii (Characiformes, Characidae) in lake Caconde, Rio Grande do Sul, Brazil. Rev. Brasil. Biol., Rio de Janeiro, 57 (2): 295-303.

IWASZKIW, J.M.; L.R. FREYRE \& E.D. SENDRA. 1983. Estudio de la maduración, época de desove y fecundidad del dentudo Oligosarcus jenynsii (Pisces, Characidae) del embalse Rio Tercero, Cordoba, Argentina. Limnobios, La Plata, 2 (7): 518-525.

MENEZES, N.A. 1969. The food of Brycon and three closely related genera the tribe Acestrorhynchini. Pap. Avulsos Zool., São Paulo, 22: 217-223.

1987. Três espécies novas de Oligosarcus Günther, 1864 e redefinição taxonômica das demais espécies do gênero (Osteichthyes, Teleostei, Characidae). Bol. Zool., São Paulo, 11: 1-39.

Moraes, M.N. DE; A.E. Ferreira; J.M. BARbosa \& A.M. Rodrigues. 1988. Aspectos da estrutura populacional do peixe-cadela Galeocharax knerii (Steindachner, 1878) (Osteichthyes, Characidae) da represa de Bariri, Rio Tietê, Estado de São Paulo. Bol. Inst. Pesca, São Paulo, 15 (2): 179: 188.

SANTOS, E.P. DOS. 1978. Dinâmica de populações aplicada à pesca e piscicultura. São Paulo, Hucitec, Edusp, 129p.

SANTOS, R.A. Dos; E.C. CAMPOS; J.J.C. DA CAMARA \& J. MANDELli JR. 1991. Curvas de maturação gonadal e crescimento de fêmeas de tambiú, Astyanax bimaculatus Linnaeus, 1758 (Characiformes, Characidae), na represa de Ibitinga, Estado de São Paulo, Brasil. Bol. Inst. Pesca, São Paulo, 18: 1-11.

SverliJ, S.B. \& A.E. Ros. 1986. El dorado, Salminus maxillosus (Pisces, Characiformes) en el rio de La Plata y rio Uruguai inferior. Rev. Invest. Des. Pesq., Lima, (6): 57-75.

VAZZOLER, A.E.A. DE M. 1981. Manual de métodos para estudos biológicos de populações de peixes. Reprodução e crescimento. Brasília, CNPq, Programa Nacional de Zoologia, 106p.

VAZZOLER, A.E.A. DE M. \& N.A. MENEZES. 1992. Síntese de conhecimentos sobre o comportamento reprodutivo dos Characiformes da América do Sul (Teleostei, Ostariophysi). Rev. Brasil. Biol., Rio de Janeiro, 52 (4): 627-640.

VAZZOLER, A.E.A. DE M. 1996. Biologia da reprodução de peixes teleósteos: teoria e prática. Maringá, EDUEM, 169p.

Wootton, R.J. 1991. Ecology of teleost fishes. London, Chapman \& Hall, 404p.

Recebido em 25.IV.1997; aceito em 17.VIII.1998. 